\title{
Lesion Severity and Hypercholesterolemia Determine Long-Term Prognosis of Vasospastic Angina Treated With Calcium Channel Antagonists
}

\author{
Masakazu Yamagishi, MD; Kenichi Ito, MD; Hiroshi Tsutsui, MD; Shunichi Miyazaki, MD; \\ Yoichi Goto, MD; Noritoshi Nagaya, MD; Tetsuya Sumiyoshi, MD*; Kenichi Fukami, MD**; \\ Kazuo Haze, MD ${ }^{\dagger}$; Masafumi Kitakaze, MD; Hiroshi Nonogi, MD; Hitonobu Tomoike, MD
}

\begin{abstract}
Although patients with medically treated vasospastic angina have a good outcome, few data exist regarding the role of underlying lesion severity associated with or without hyperlipidemia in the prognosis. Therefore, the aim of the present study was to assess the relationship between the long-term outcome of vasospastic angina and the factors influencing its prognosis. A total of 256 patients (219 men, 37 women; mean age, 54.1 \pm 9.2 ) who had coronary spasm with or without underlying lesions and were being treated with calcium channel antagonists were enrolled and followed for 13.6 \pm 3.7 years. Cardiac events consisted of cardiac death and ischemic events, which included acute myocardial infarction and unstable angina. Cox analysis selected coronary artery stenosis (CAS, $\geq 50 \%$ ) and risk factors such as age, hypertension, diabetes mellitus, low-density lipoprotein-cholesterol (LDL-C), sex and smoking. There were 19 cases of cardiac death (7.4\%) and 58 of ischemic events (22.7\%) during the follow-up period. The presence of significant CAS was an independent predictor of event-free survival (hazard ratio $(\mathrm{HR})=2.84,95 \%$ confidence interval $(\mathrm{CI})=1.79-4.52, \mathrm{p}<0.0001$ ). In 193 patients without significant CAS, there were 10 cases of cardiac death $(5.2 \%, \mathrm{p}<0.05)$ and 34 of ischemic events $(17.6 \%, \mathrm{p}<0.01)$. In that group, high LDL-C was the independent predictor of event-free survival $(\mathrm{HR}=3.89,95 \% \mathrm{CI}=1.20-12.6$, $\mathrm{p}=0.02$ ). Kaplan-Meier survival analysis revealed significantly lower event-free survival in patients with than in those without lesions $(\mathrm{p}<0.0001$ by log-rank test). These results demonstrate that the most important factor for long-term prognosis of vasospastic angina treated with calcium channel antagonists is significant CAS. High LDL-C, which might alter the underlying coronary endothelial function and/or accelerate atherosclerotic lesions, could also contribute to the occurrence of cardiac events, particularly in patients without significant CAS. (Circ J 2003; 67: 1029-1035)
\end{abstract}

Key Words: Calcium channel antagonist; Coronary artery disease; Long-term prognosis; Vasospasm

C oronary spasm provokes the myocardial ischemia associated with angina pectoris, acute myocardial infarction and sudden death!, ${ }^{1,2}$ Calcium channel antagonists, nitrates or a combination of both drugs have been used effectively to prevent coronary spasm $3^{3-6}$ Indeed, the overall cardiac mortality is relatively low and the prognosis seems to be good for medically treated patients with coronary spasm? ${ }^{7}$ Coronary spasm frequently occurs in minimally narrowed coronary segments, $, 10,11$ suggesting a pathophysiologic correlation between coronary spasm and atherosclerosis, which has been demonstrated in an experimental animal model $!^{2}$ Several studies have examined the relationship between the clinical characteristics and prognosis of this type of angina, and demonstrated that preexist-

(Received April 30, 2003; revised manuscript received September 24, 2003; accepted September 26, 2003)

Division of Cardiovascular Medicine, National Cardiovascular Center, Suita, *Division of Cardiology, Sakakibara Heart Institute, Tokyo, **Division of Cardiology, Iwate Medical College, Morioka and Division of Cardiology, Osaka City General Hospital Medical Center, Osaka, Japan

A part of this work was presented at American Heart Association Scientific Sessions in Anaheim, 2001.

Mailing address: Masakazu Yamagishi, MD, PhD, FACC, Division of Cardiovascular Medicine, National Cardiovascular Center, 5-7-1 Fujishiro-dai, Suita, Osaka 565-8565, Japan. E-mail: myamagi@ hsp. ncvc.go.jp ing atherosclerosis could be an important risk factor for cardiac death and myocardial infarction during the relatively early phase ${ }^{5-9}$ However, there is little information regarding the long-term prognosis of medically treated coronary spasm with or without atherosclerotic risks, such as underlying coronary artery stenosis (CAS) and intrinsic hyperlipidemia. Such data should be important for not only preventing vasospasm, but also preventing the development of atherosclerosis. The purpose of this study was, first, to determine the event-free survival rate in patients with angiographically documented coronary spasm, and second, to identify the clinical predictors of cardiac events, particularly in patients with and without significant CAS.

\section{Methods}

\section{Patient Population}

Of 2,740 patients who underwent diagnostic coronary angiography for suspected ischemic heart disease between 1977 and 1987, 284 consecutive patients with coronary spasm were enrolled and followed. During the follow-up, 21 patients went missing. Patient enrollment was completed in 1987 when 3-hydroxy-3-methyleglutaryl coenzyme A (HMG-CoA) reductase inhibitor, which might have altered the long-term prognosis, ${ }^{13}$ was not generally available. Therefore, no patient had been given HMG-CoA reductase 
Table 1 Baseline Clinical and Angiographic Characteristics

\begin{tabular}{lcccc}
\hline \hline & $\begin{array}{c}\text { Total } \\
(n=256)\end{array}$ & $\begin{array}{c}\text { Without CAS } \\
(n=193)\end{array}$ & $\begin{array}{c}\text { With CAS } \\
(n=63)\end{array}$ & $p$ value \\
\hline Age (years) & $54.1 \pm 9.2$ & $54.4 \pm 8.0$ & $55.0 \pm 10.3$ & $N S$ \\
Male sex (\%) & $219(85.5)$ & $163(84.5)$ & $56(88.9)$ & $N S$ \\
Coronary risk factors & & & & \\
Hypertension (\%) & $115(44.9)$ & $91(47.2)$ & $24(38.0)$ & $N S$ \\
High LDL-C (\%) & $61(23.8)$ & $39(20.2)$ & $22(34.9)$ & $N S$ \\
Diabetes mellitus (\%) & $7(2.7)$ & $3(1.6)$ & $4(6.3)$ & $N S$ \\
Smoking (\%) & $174(66.8)$ & $129(66.8)$ & $45(71.4)$ & $N S$ \\
Arrhythmia during angina (\%) & $10(3.9)$ & $10(5.2)$ & $0(0)$ & $N S$ \\
Multivessel spasm (\%) & $18(7.0)$ & $17(8.8)$ & $1(1.6)$ & $N S$ \\
\hline
\end{tabular}

CAS, coronary artery stenosis; $L D L-C$, low-density lipoprotein-cholesterol.

Table 2 Incidence of Cardiac Events During Follow-up Period

\begin{tabular}{lcccc}
\hline \hline Cardiac event & $\begin{array}{c}\text { Total } \\
(n=256)\end{array}$ & $\begin{array}{c}\text { Without CAS } \\
(n=193)\end{array}$ & $\begin{array}{c}\text { With CAS } \\
(n=63)\end{array}$ & p value \\
\hline Cardiac death (\%) & $19(7.4)$ & $10(5.2)$ & $9(14.3)$ & 0.017 \\
Sudden death $(\%)$ & $8(3.1)$ & $3(1.6)$ & $5(7.9)$ & 0.024 \\
Myocardial infarction (\%) & $3(1.1)$ & $2(1.0)$ & $1(1.6)$ & $N S$ \\
Heart failure (\%) & $5(2.0)$ & $4(2.1)$ & $1(1.6)$ & $N S$ \\
Others (\%) & $3(1.2)$ & $1(0.5)$ & $2(3.2)$ & $N S$ \\
Ischemic event (\%) & $58(22.7)$ & $34(17.6)$ & $24(38.1)$ & $<0.01$ \\
Nonfatal myocardial infarction (\%) & $15(5.9)$ & $8(4.1)$ & $7(11.1)$ & 0.041 \\
Unstable angina (\%) & $29(11.3)$ & $21(10.9)$ & $8(12.7)$ & $N S$ \\
PTCA (\%) & $12(4.7)$ & $5(2.6)$ & $7(11.1)$ & 0.011 \\
CABG (\%) & $2(0.8)$ & $0(0)$ & $2(3.2)$ & $N S$ \\
Time of event from registration (years) & $4.9 \pm 4.7$ & $4.6 \pm 4.3$ & $5.3 \pm 5.3$ & $N S$ \\
\hline
\end{tabular}

$C A B G$, coronary artery bypass grafting; CAS, coronary artery stenosis; PTCA, percutaneous transluminal coronary angioplasty.

inhibitor at the time follow-up began, but 7 patients were unexpectedly given this drug during the follow-up period, particularly after 1990 . Therefore, these 28 patients were excluded, and a total of 256 patients were analyzed in this

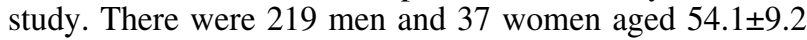
years. All the patients had chest pain at rest and/or on exertion. ECG changes such as ST elevation or depression during chest pain attacks were demonstrated in 154 patients.

\section{Cardiac Catheterization Procedures}

Written informed consent was obtained from all patients for cardiac catheterization and the provocation of coronary spasm. All patients were fasted and received 3,000-5,000 U heparin intravenously before the procedure. Calcium channel antagonists, nitrates, $\beta$-blockers and other anti-anginal drugs were discontinued at least $9 \mathrm{~h}$ before procedures, because these drugs could alter the basal coronary tone associated with the occurrence of spasm.

After control coronary angiography, the provocative tests were performed. We initially used intravenous ergonovine to induce spasm ${ }^{14}$ but since 1986 , the intracoronary ergonovine test has been used for safety reasons. ${ }^{15}$ When coronary spasm was not provoked, ergonovine maleate was administered until the total dose reached $0.4 \mathrm{mg}$ in the intravenous test, or $0.04 \mathrm{mg}$ in the intracoronary test. Under these conditions, the standard 12-lead ECG was continuously monitored to record ST change $(\geq 0.1 \mathrm{mV})$ and associated arrhythmias. When chest pain or significant ST-segment changes were observed, selective coronary angiography was immediately performed. Coronary spasm was considered positive when there was luminal narrowing $\geq 75 \%$; however, diffuse spasm $\geq 75 \%$ without signs of myocardial ischemia was not considered positive, because it is difficult to differentiate real spasm from a pharmacological reaction.6 Multi-vessel spasm was defined as vasospasm in more than one major coronary artery.

After provocation, $0.25-0.5 \mathrm{mg}(0.5 \mathrm{mg} / \mathrm{ml})$ nitroglycerin was given to obtain maximal coronary dilation, and coronary angiography was again performed in multiple projections to evaluate the extent and severity of CAS. Left ventriculography was performed to calculate the left ventricular ejection fraction by the area-length method. Coronary angiography and left ventriculography were evaluated by at least 2 angiographers who were independent of each other. Significant CAS was defined as lumen stenosis $\geq 50 \%$. In the case of repeated angiography, progression of lesion severity was defined as an increase of $\geq 20 \%$ in a preexisting stenosis of $\geq 50 \%$, and an increase of $\geq 30 \%$ in a stenosis $<50 \%$, or any increase in lesion severity that resulted in total coronary occlusion. New stenoses were defined as stenoses $\geq 20 \%$ that developed at a site that was previously angiographically normal! ${ }^{17}$

\section{Assessment of Coronary Risk Factors}

The coronary risk factors at baseline were hypertension, diabetes mellitus, high low-density lipoprotein-cholesterol (LDL-C) and history of smoking. Blood sugar, total cholesterol, LDL-C, high-density lipoprotein-cholesterol, and triglycerides were measured on the morning of angiography while the patient was still in a fasting state. In patients who could be followed up for more than 6 months, these variables were re-examined as frequently as possible during the follow-up period. In patients in whom LDL-C had not been measured and the triglyceride concentration was $<300 \mathrm{mg} / \mathrm{dl}$, the LDL-C concentration was calculated using the following equation: $\mathrm{LDL}-\mathrm{C}=($ total cholesterol - 


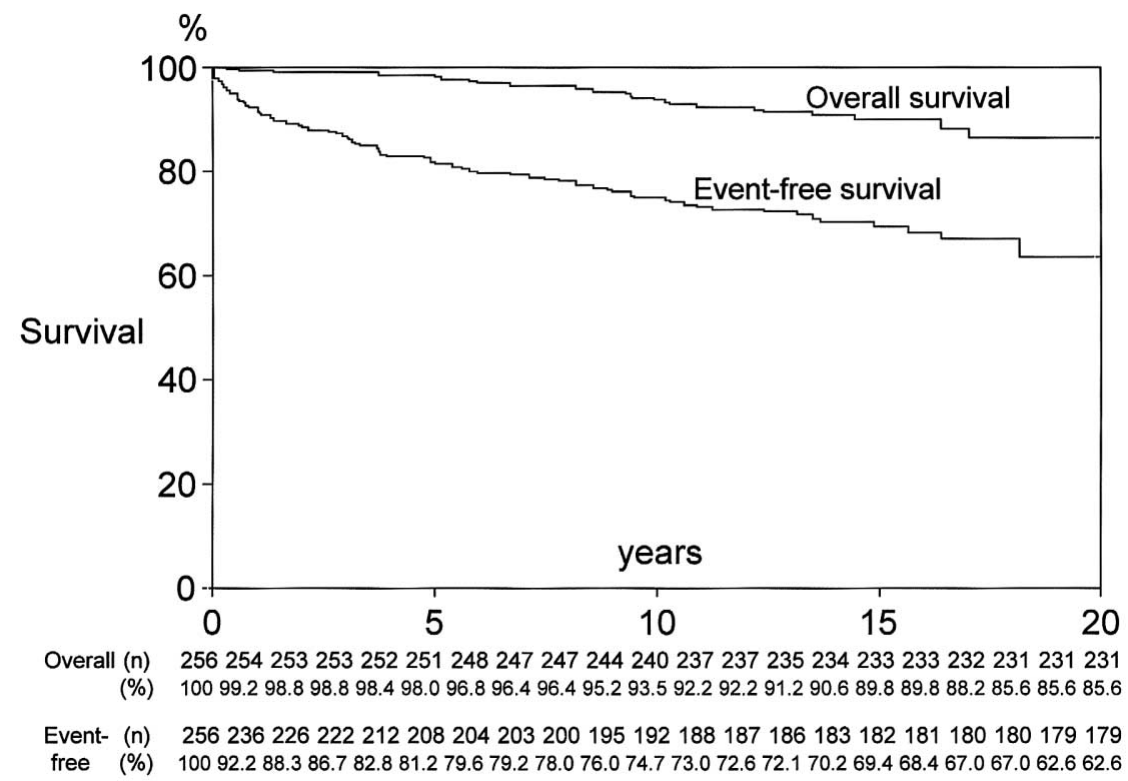

Fig 1. Kaplan-Meier analysis of overall mortality and event-free survival rate for entire patient group. Vertical and horizontal axes represent $\%$ survival rate and time from registration, respectively.
HDL-C-1/5 triglycerides). Hypertension was defined as systolic blood pressure $>140 \mathrm{mmHg}$, diabetes mellitus was defined as fasting glucose $>140 \mathrm{mg} / \mathrm{dl}$, and high LDL-C was defined as serum LDL-C concentration $>140 \mathrm{mg} / \mathrm{dl}$. A smoking history was obtained from all patients: smokers were defined as patients who had smoked more than 20 cigarettes per day for $>20$ years. We also evaluated the contribution of the patients' sex and age to the prognosis.

\section{Long-Term Follow-up}

The cardiac events included cardiac death and ischemic events. Cardiac death consisted of sudden death, which was defined as death within $1 \mathrm{~h}$ after collapse, and death associated with acute myocardial infarction, heart failure, or other cardiovascular disease. Ischemic events consisted mainly of non-fatal myocardial infarction, unstable angina and the need for percutaneous transluminal angioplasty (PTCA) or coronary artery bypass surgery (CABG). Unstable angina was defined as worsening of chest symptoms, ${ }^{18}$ and except for emergency procedures the indication for PTCA and CABG was considered by at least 2 cardiologists independent of this study.

Myocardial infarction was diagnosed on the basis of the development of a new Q wave in the ECG and elevation of serum concentrations of cardiac enzymes. Unstable angina was defined as repeated anginal attacks at rest. Non-fatal myocardial infarction was defined as survival of the patient who could then be discharged from hospital. In this study, if a patient died of cardiac death and had suffered an ischemic event before death, the cardiac event was not defined as cardiac death, but as an ischemic event. Accordingly, a cardiac event in this study meant the first cardiac event that occurred during follow-up.

All patients were given at least either dihydropyridine or diltiazem hydrochloride. Drug therapy was started immediately after angiographic diagnosis and continued by the individual physicians until the end-point. The end-points of follow-up were the first cardiac event and non-cardiac death without a cardiac event. The follow-up period of each patient was calculated from the date of the initial diagnostic angiography. Those who did not visit for follow-up examination and checking the status of medical compliance were
Table 3 Predictors of Cardiac Events in All Patients During Follow-up Period

\begin{tabular}{lccc}
\hline \hline & Hazard ratio & $95 \%$ CI & p value \\
\hline CAS & 2.84 & $1.79-4.52$ & $<0.0001$ \\
High LDL-C & 2.21 & $0.79-6.21$ & 0.079 \\
Diabetes mellitus & 1.72 & $0.94-3.16$ & 0.16 \\
Hypertension & 1.21 & $0.71-2.00$ & 0.45 \\
Sex & 1.18 & $0.59-2.37$ & 0.65 \\
Age & 0.99 & $0.97-1.03$ & 0.89 \\
Smoking & 0.99 & $0.58-1.68$ & 0.96 \\
\hline
\end{tabular}

CAS, coronary artery stenosis; $C I$, confidence interval; $L D L-C$, low-density lipoprotein-cholesterol.

followed by telephone interviews with the patient or the family, from whom the status of smoking was carefully elicited. When anginal attacks became frequent or were not relieved by medical treatment, the patient was admitted and coronary angiography was performed again to determine alternative methods of management.

\section{Statistical Analysis}

All values are expressed as mean \pm SD. Continuous variables were compared by unpaired Students' t-test. Categorical variables were compared by chi-square test (with Fisher's exact test, as appropriate for smaller sample size). Multivariate analysis with Cox proportional-hazards regression analysis was used to assess the independent significance of prognostic factors for cardiac events. Event-free survival rate was calculated using the Kaplan-Meier method, and differences between survival curves were assessed by the log-rank test $1^{9}$ Values of $\mathrm{p}<0.05$ were considered significant. Analyses were performed using StatView 4.5 statistical software (Abacus Concepts, Berkeley, CA, USA).

\section{Results}

Baseline Data Collection (Table 1)

Coronary spasm was angiographically demonstrated during spontaneous angina in 40 patients and in 216 patients by provocation tests. Spasm in more than one 


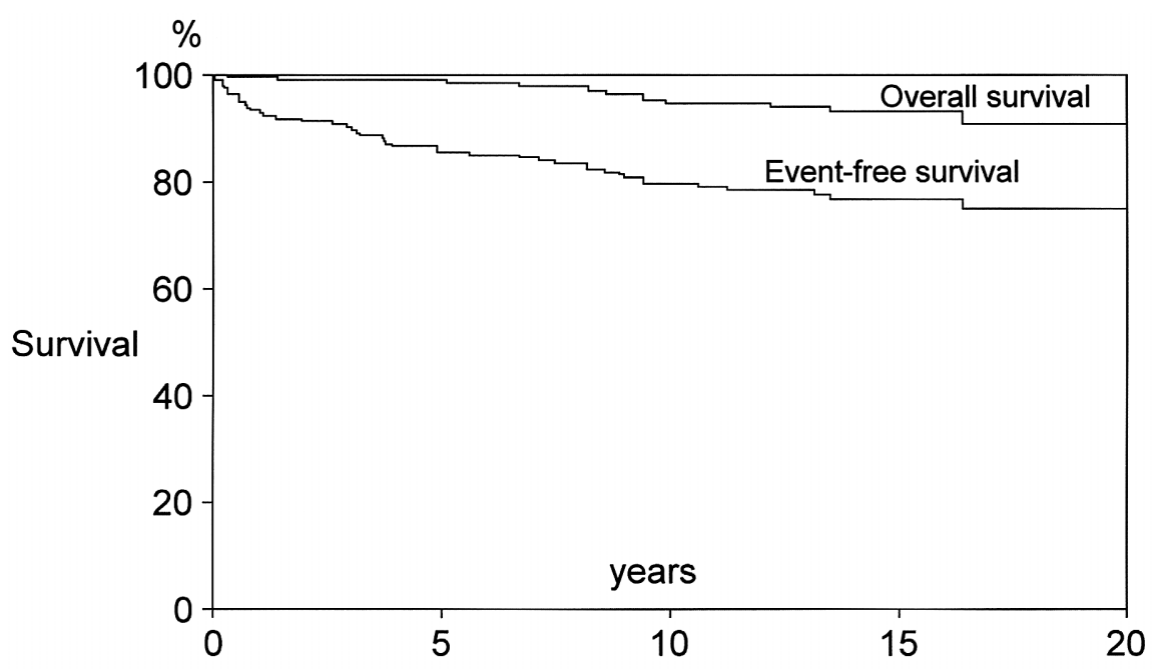

Overall (n) 193192191191191191190189189186183183183182181181181180180180180 (\%) 10099.599 .099 .099 .099 .098 .497 .997 .996 .394 .694 .694 .693 .993 .093 .093 .090 .890 .890 .890 .8

Event- (n) 193180176174167165164163161156154153152152150150150149149149149 free (\%) 10093.391 .290 .286 .585 .585 .084 .483 .480 .779 .679 .078 .578 .576 .776 .776 .774 .674 .674 .674 .6
Fig 2. Kaplan-Meier analysis of overall mortality and event-free survival in patients without significant coronary artery stenosis. Vertical and horizontal axes represent \% survival rate and time from registration, respectively.
Table 4 Predictors of Cardiac Events in Patients Without Significant CAS During Follow-up Period

\begin{tabular}{lccc}
\hline \hline & Hazard ratio & $95 \%$ CI & p value \\
\hline High LDL-C & 3.89 & $1.20-12.6$ & 0.02 \\
Sex & 1.51 & $0.64-3.59$ & 0.35 \\
Smoking & 1.14 & $0.57-2.28$ & 0.71 \\
Age & 0.99 & $0.95-1.03$ & 0.51 \\
Hypertension & 0.95 & $0.50-1.82$ & 0.87 \\
Diabetes mellitus & 0.97 & $0.13-7.41$ & 0.98 \\
\hline
\end{tabular}

$C A S$, coronary artery stenosis; $C I$, confidence interval; $L D L-C$, low-density lipoprotein-cholesterol.

major coronary artery was demonstrated in 18 patients. Of the 256 patients, 63 had significant CAS; 53 with singlevessel disease, 8 with double-vessel disease, and 2 with triple-vessel disease. Among the remaining 193 patients who did not have significant CAS, 99 exhibited normal coronary angiography after nitroglycerin administration.

ECG during anginal attacks showed ST elevation in 123 patients, depression in 51 patients and no significant ST change in 82 patients. Ventricular tachycardia was recorded in 6 patients, and second- or third-degree atrioventricular block in 4 patients during provoked coronary spasm. Left ventriculography was normal in 200 patients; segmental wall motion abnormalities were observed in 31 patients. Left ventriculography was not performed in 25 patients because previous echocardiography had shown normal wall motion and cardiac systolic function. Ninety-nine patients were treated with a calcium channel antagonist such as dihydropyridine (30-60 mg/day) or diltiazem hydrochloride $(90-120 \mathrm{mg} /$ day), and 157 patients with a combination of calcium channel antagonist and nitrate $(40-80 \mathrm{mg} / \mathrm{day})$. In addition to these medications, $\beta$-blockers were used in 9 patients. The dose of each drug was calculated to prevent the occurrence of angina during daily activities.

\section{Incidence of Cardiac Events During Follow-up (Table 2)}

The mean duration of follow-up was $13.6 \pm 3.7$ years (range 0.3-20 years). The first cardiac event occurred at $4.9 \pm 4.7$ years from registration. Of the 46 patients who died during follow-up, 19 died of cardiac death (8 sudden death, 3 myocardial infarction, 5 unexpected heart failure, 3 unknown cause). The other 27 patients died from noncardiac causes. Ischemic events occurred in 58 patients (non-fatal acute myocardial infarction in 15, unstable angina in 29, PTCA in 12, CABG in 2). The coronary lesions causing the ischemic events were confirmed to coincide with the previous sites of vasospasm in 30 patients and could not be confirmed in the remaining 28 patients.

The overall survival rate was $99.2 \%$ at 1 year, $98.0 \%$ at 5 years, $93.5 \%$ at 10 years and $85.6 \%$ at 20 years. Under these conditions, the event-free survival rate was $92.2 \%$, $81.2 \%, 74.7 \%$, and $62.6 \%$, respectively (Fig 1). When all of the 7 variables were analyzed, the presence of CAS was shown to be an independent predictor of cardiac events (hazard ratio $(\mathrm{HR})=2.84,95 \%$ confidence interval $(\mathrm{CI})=$ $1.79-4.52, \mathrm{p}<0.0001)$. There was no difference in survival rate between the patients with CAS in the left coronary and right coronary arteries. High LDL-C and diabetes mellitus had relatively high hazard ratios (2.21 and 1.72 , respectively) without statistical significance. Other factors were not significant predictors of cardiac events (Table 3 ).

In patients without CAS, Kaplan-Meier survival analysis revealed an event-free survival rate of $85.5 \%$ at 5 years, $79.6 \%$ at 10 years, $76.7 \%$ at 15 years and $74.6 \%$ at 20 years (Fig 2). Under these conditions, high LDL-C was the only independent predictor of event-free survival $(\mathrm{HR}=3.89$, 95\% CI $=1.20-12.6, \mathrm{p}=0.02$, Table 4$)$. As for the relationship between ST deviation and occurrence of cardiac events, cardiac events occurred in 56 of 174 patients (32.2\%) with ST deviation, elevation or depression, during vasospasm and in 21 of 82 patients $(25.6 \%$, NS) without ST deviation. In 99 patients with normal angiography after nitroglycerin, the incidence of cardiac events and the eventfree survival rate were not different from those of the remaining 94 patients with minimal and not significant lesion $(\mathrm{p}=0.074)$.

\section{Association Between CAS and Cardiac Events}

Although there were no differences in the baseline clinical characteristics between patients with and without CAS 


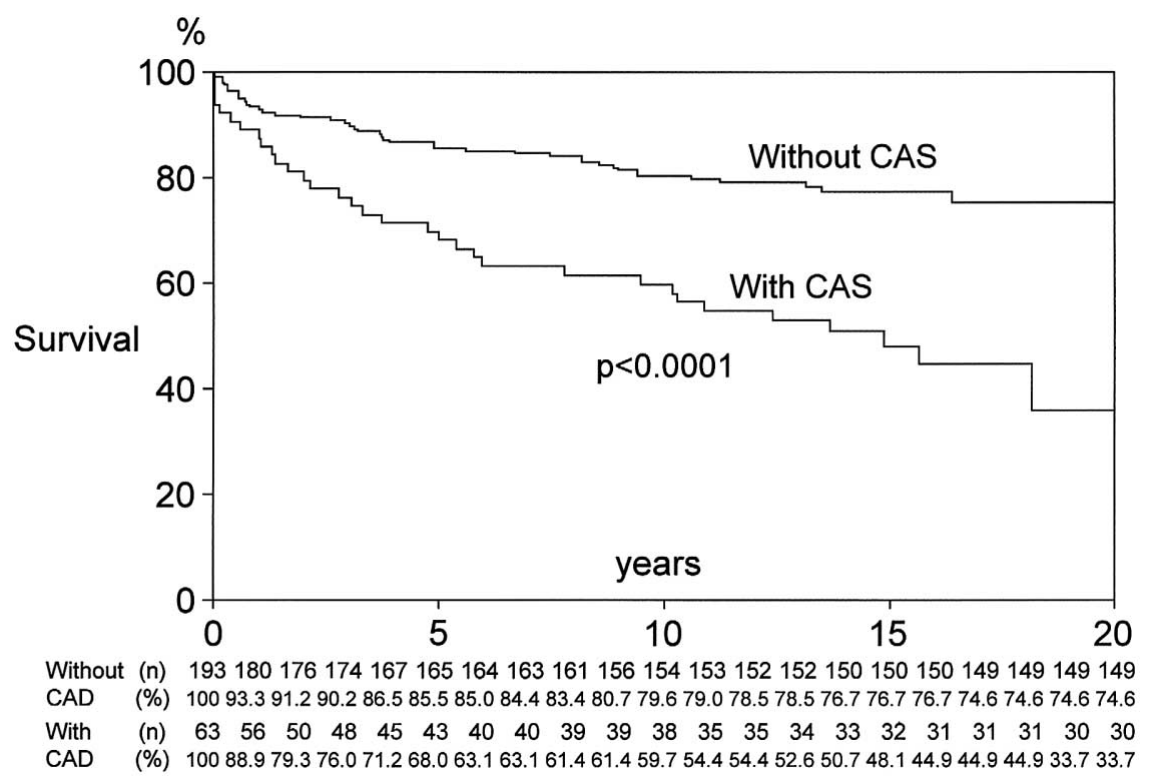

Fig 3. Comparison of event-free survival curves between patients with and without significant coronary artery stenosis (CAS). Vertical and horizontal axes represent \% survival rate and time from registration, respectively.
(Table 1), the overall rate of cardiac events was significantly lower in patients without CAS $(22.8 \%)$ than in those with CAS $(52.4 \%, \mathrm{p}<0.01)$. As for cardiac deaths, 9 of 63 patients with CAS died and only 10 of 193 patients without CAS, yielding a significant difference in cardiac mortality between patients with and without CAS ( $\mathrm{p}=0.017)$. Also, there was a significant difference in the incidence of sudden death between these subgroups $(\mathrm{p}=0.024)$.

Eight of 15 patients with non-fatal myocardial infarction did not have CAS whereas the remaining 7 patients did. Thus, the incidence of non-fatal myocardial infarction in patients without CAS was significantly lower than that in patients with CAS $(\mathrm{p}=0.041)$. However, there was no significant difference in the time interval between registration and the first cardiac events in patients without $(4.6 \pm 4.3$ years) and with $(5.3 \pm 5.3$ years) CAS. There was a marked difference in the event-free survival rate between the subgroups with and without CAS ( $<<0.0001$, Fig 3 ).

Coronary angiography was repeated in 45 of the 256 patients. Progression of coronary atherosclerosis was observed in 10 patients with CAS $(45.5 \%)$ and in 13 patients without CAS $(56.5 \%, \mathrm{NS})$. It was interesting that progression of coronary atherosclerosis was more frequently observed at previous sites of spasm (17 patients or $37.8 \%$ ) than at nonspastic sites ( 7 patients or $15.5 \%, \mathrm{p}=0.016$ ).

\section{Discussion}

\section{Prognostic Value of CAS and High LDL-C}

One of the important findings of the present study is that the presence of significant CAS was an independent predictor of event-free survival, as well as the simple survival, in patients with vasospastic angina treated with calcium channel antagonists. The local effect of more complete occlusion during coronary spasm may predispose to malignant arrhythmia or severe myocardial ischemia, although there was no difference in the incidence of cardiac events, including sudden death, in patients with and without ST deviation that may represent the severity of transient myocardial ischemia. Treatment with calcium channel antagonists could reduce this transient ischemia in both patients with and without significant CAS.
Coronary spasm is a possible mechanism for the progression of atherosclerosis! ${ }^{7,20,21}$ Indeed, in the present study repeated coronary angiography revealed that approximately $40 \%$ of patients had progression of coronary atherosclerosis at the sites of previously demonstrated vasospasm. We speculate that coronary spasm accelerates the progression of atherosclerosis, resulting in sudden death and other cardiac events. Under these conditions, elevation of the LDL-C concentration might play an important role in aggravating atherosclerosis through the disintegration of endothelial function.22 However, high LDL-C was second to CAS in terms of hazard ratio in all the present patients. It is possible that a relatively close relation of high LDL-C to significant CAS might make it difficult to differentiate LDL-C as an independent factor in the presence of CAS. Actually, this was shown to be the most important independent factor for cardiac events in patients without CAS. Based on an animal model, an important early step in the development of atherosclerotic lesions is endothelial dysfunction associated with high cholesterol, leading to abnormal vessel tonus characterized by paradoxical constriction to physiologic and pathologic stimuli23 Vasospasm may be an extreme of this phenomenon and related to lesion development. From this point of view, it is interesting to speculate that high LDL-C may be related to impairment of coronary endothelial dysfunction 24 although recent work does not support this hypothesis. 25

Previous studies suggest that the occurrence of coronary spasm is independent of plasma cholesterol concentrations; in the present study, two-thirds of the patients had normal cholesterol levels. However, under treatment with calcium channel antagonists, which effectively prevent spasm, conventional risk factors such as hypercholesterolemia might be important for the development of atherosclerotic lesions associated with cardiac events. Also it was unclear in the previous studies whether or not the LDL-C concentration was high, even though the total cholesterol concentration was within normal range.

The finding that the incidence of sudden death was lower than in previous studies may be explained by the small number of patients with life-threatening arrhythmias in the present patient group; Millar et al reported that nearly $80 \%$ 
of patients who died from sudden death had a history of ventricular tachyarrhythmias? ${ }^{26}$ Even so, 8 patients died suddenly during the follow-up period. It is unclear whether the presence of CAS is a major determinant of sudden death, because sudden death also occurred frequently in patients without $\mathrm{CAS}^{27}$ Although a previous study suggested that spasm in multiple vessels could be an important determinant of prognosis 28 the present study could not confirm this finding because of the small number of patients with multi-vessel spasm. One of the reasons why there were relatively few instances of multi-vessel spasm was the different way of provoking spasm. Selective intracoronary injection of ergonovine might not have induced multivessel spasm in some patients in the present study.

\section{Prognostic Value of Other Risk Factors}

Except for high LDL-C, there was no statistically significant difference between the other coronary risk factors and cardiac events, although smoking is considered to be one of the major risk factors for the occurrence of vasospastic angina $2^{2}$ The patients with a history of smoking were carefully questioned about whether they had discontinued smoking during the follow-up period, but it is difficult to confirm the actual status of smoking just by questionnaires at the out-patient clinic. Discontinuation or, at least, a reduction in smoking might result in statistical insignificance of the history of smoking as a risk factor for cardiac events and sudden death.

Coronary spasm without CAS has a fairly good longterm clinical outcome, but even so, high LDL-C was an independent prognostic factor in this subgroup. This suggests that for patients with coronary spasm, manipulation of the therapeutic regimen to prevent not only the occurrence of vasospasm, but also the progression of atherosclerosis should be considered. Calcium channel antagonists such as nifedipine or nicardipine do not reduce cardiac events $^{30,31}$ and therefore, a combination with other agents, such as an HMG-CoA reductase inhibitor, that may stabilize the atherosclerotic plaque would further reduce cardiac events in vasospastic angina, ${ }^{13}$ although the present study excluded patients in whom HMG-CoA reductase inhibitors were given.

The finding that other traditional risk factors were not significant for long-term prognosis can be explained by the specific situation of vasospastic angina. Indeed, previous studies demonstrated that coronary spasm could occur in the absence of hypertension and diabetes mellitus ${ }^{29}$ Magnesium deficiency, which was not determined in the present study, might be another risk factor for prognosis of coronary spasm, because $50 \%$ of patients with recent myocardial infarction have been shown to have coronary spasm associated with it?2

\section{Study Limitations}

We previously reported that even in the absence of angiographic coronary disease, atherosclerosis can be demonstrated by intravascular ultrasound at the sites of spasm 33,34 Therefore, we could not precisely evaluate the progression or regression of coronary disease in all patients. Prospective follow-up of patients using ultrasound may resolve this problem. Whether patients consistently complied with medication during the follow-up period is important in considering the long-term prognosis. However, the mid-term prognosis of the present study was similar to those of others, 8 which suggests that there was accepta- ble drug compliance during the follow-up period. The use of different kinds of calcium channel antagonists for treatment may have had a small effect on the long-term prognosis, although dihydropyridines and diltiazem have a similar effect on coronary spasm in the relatively acute phase ${ }^{3-6}$

Although it is clinically interesting to consider the relationship between the frequency of anginal attacks and longterm prognosis, we could not determine the significance, because of difficulty in quantitatively estimating the frequency. For the same reason, it was also difficult to correlate the presence of CAS with anginal frequency.

To further understand the role of high LDL-C in the long-term prognosis of vasospastic angina, the medical regimen of HMG-CoA reductase inhibitors in addition to calcium channel antagonists should be challenged. It is also necessary to compare the long-term prognosis between CAS patients with and without spasm. However, practically, it is difficult to enroll stable patients without coronary spasm, because PTCA or CABG rather than medical treatment is now the primary treatment 35

Finally, the present study dealt only with Japanese patients with coronary spasm. However, despite the racial difference in coronary vasomotor reactivity, ${ }^{36}$ the clinical outcome of the present patients is similar to the results of studies in Europe and North America. Therefore, the present data provide important information on the long-term prognosis of medically treated coronary spasm patients with or without CAS.

\section{Acknowledgments}

We thank Mr Nobuo Shirahashi, MS, for his assistance with the statistical analysis.

This work was supported in part by grants from the Osaka Heart Club, Osaka (to M.Y.), from Daiwa Securities Healthcare Foundation, Tokyo (to M.Y.), and by grants from the Ministry of Health, Welfare and Labor of Japan, Tokyo (to M.K. and H.T.).

\section{References}

1. Hillis LD, Braunwald E. Coronary-artery spasm. N Engl J Med 1978; 299: $695-702$

2. Maseri A, Severi S, Nes MD, L'Abbate A, Chierchia S, Marzilli M, et al. 'Variant' angina: One aspect of a continuous spectrum of vasospastic myocardial ischemia. Am J Cardiol 1978; 42: 1019-1035.

3. Schroeder JS, Lamb IH, Bristow MR, Ginsburg R, Hung J, McAuley BJ. Prevention of cardiovascular events in variant angina by longterm diltiazem therapy. J Am Coll Cardiol 1983; 1: 1507-1511.

4. Bott-Silverman C, Heupler FA. Natural history of pure coronary artery spasm in patients treated medically. J Am Coll Cardiol 1983; 2: $200-205$.

5. Waters DD, Miller DD, Szlachcic J, Bouchard A, Methe M, Kreeft J, et al. Factors influencing the long-term prognosis of treated patients with variant angina. Circulation 1983; 68: 258-265.

6. Walling A, Waters DD, Miller DD, Roy D, Pelletier GB, Theroux P. Long-term prognosis of patients with variant angina. Circulation 1987; 76: 990-997.

7. Nakamura M, Takeshita A, Nose Y. Clinical characteristics associated with myocardial infarction, arrhythmias, and sudden death in patients with vasospastic angina. Circulation 1987; 75: 1110-1116.

8. Yasue H, Takizawa A, Nagao M, Nishida S, Horie M, Kubota J, et al. Long-term prognosis for patients with variant angina and influential factors. Circulation 1988; 78: 1-9.

9. Shimokawa H, Nagasawa K, Irie T, Egashira S, Egashira K, Sagara $\mathrm{T}$, et al. Clinical characteristics and long-term prognosis of patients with variant angina: A comparative study between western and Japanese populations. Int J Cardiol 1988; 18: 331-349.

10. Heupler FA. Syndrome of symptomatic coronary arterial spasm with nearly normal coronary angiograms. Am J Cardiol 1980; 45: 873881.

11. Mercurio P, Kronzon I, Winer H. Spasm of normal or minimally narrowed coronary artery in the presence of severe fixed stenoses of the remaining vessels: Clinical and angiographic observations. 
Circulation 1982; 65: 825-830

12. Shimokawa H, Tomoike H, Nabeyama S, Yamamoto H, Ishii Y, Tanaka K, et al. Coronary artery spasm induced in miniature swine: Angiographic evidence and relation to coronary atherosclerosis. Am Heart J 1985; 110: 300-310.

13. Jukema JW, Bruschke AV, van Boven AJ, Reiber JH, Bal ET, Zwinderman $\mathrm{AH}$, et al. Effect of lipid lowering by pravastatin on progression and regression of coronary artery disease in symptomatic men with normal to moderately elevated serum cholesterol levels: The Regression Growth Evaluation Statin Study (REGRESS). Circulation 1995; 91: 2528-2540.

14. Schroeder JS, Bolen JL, Quint RA, Clark DA, Hayden WG, Higgins $\mathrm{CB}$, et al. Provocation of coronary spasm with ergonovine maleate: New test with results in 57 patients undergoing coronary arteriography. Am J Cardiol 1977; 40: 487-491.

15. Hackett D, Larkin S, Chierchia S, Davies G, Kaski JC, Maseri A. Induction of coronary artery spasm by a direct local action of ergonovine. Circulation 1987; 75: 577-582.

16. Miyao $\mathrm{Y}$, Kugiyama $\mathrm{K}$, Kawano $\mathrm{H}$, Motoyama $\mathrm{T}$, Ogawa H, Yoshimura M, et al. Diffuse intimal thickening of coronary arteries in patients with coronary spastic angina. J Am Coll Cardiol 2000; 36: $432-437$.

17. Kaski JC, Tousoulis D, McFadden E, Crea F, Pereira WI, Maseri A Variant angina pectoris: Role of coronary spasm in the development of fixed coronary obstructions. Circulation 1992; 85: 619-626.

18. Chahine RA. Unstable angina: The problem of definition. Br Heart J 1975; 37: 1246-1249.

19. Glantz SA. How to analyze survival data. In: Primer of biostatistics. New York: McGraw-Hill; 1997; 373-402.

20. Nobuyoshi M, Tanaka M, Nosaka H, Kimura T, Yokoi H, Hamasaki $\mathrm{N}$, et al. Progression of coronary atherosclerosis: Is coronary spasm related to progression? J Am Coll Cardiol 1991; 18: 904-910.

21. Suzuki H, Kawai S, Aizawa T, Kato K, Sunayama S, Okada R, et al. Histological evaluation of coronary plaque in patients with variant angina: Relationship between vasospasm and neointimal hyperplasia in primary coronary lesions. J Am Coll Cardiol 1999; 33: 198-205.

22. Egashira K, Tomoike H, Yamamoto Y, Yamada A, Hayashi Y, Nakamura M. Histamine-induced coronary spasm in regions of intimal thickening in miniature pigs: Roles of serum cholesterol and spontaneous or induced intimal thickening. Circulation 1986; 74: $826-837$.

23. Kuga T, Tagawa H, Tomoike H, Mitsuoka W, Egashira S, Ohara Y, et al. Role of coronary artery spasm in progression of organic coronary stenosis and acute myocardial infarction in a swine model: Importance of mode of onset and duration of coronary artery spasm. Circulation 1993; 87: 573-582.

24. Moriyama Y, Tsunoda R, Harada M, Miyao Y, Yoshimura M,
Kugiyama K, et al. Nitric oxide-mediated vasodilatation is decreased in forearm resistance vessels in patients with coronary spastic angina. Jpn Circ J 2001; 65: 81 -86.

25. Fukuda Y, Teragawa H, Matsuda K, Yamagata T, Matsuura H, Chayama K. Tetrahydrobiopterin improves coronary endothelial function, but does not prevent coronary spasm in patients with vasospastic angina. Circ J 2002; 66: 58-62.

26. Miller DD, Waters DD, Szlachcic J, Theroux P. Clinical characteristics associated with sudden death in patients with variant angina. Circulation 1982; 66: 588-592.

27. MacAlpin RN. Cardiac arrest and sudden unexpected death in variant angina: Complications of coronary spasm that can occur in the absence of severe organic coronary stenosis. Am Heart J 1993; 125: $1011-1017$.

28. Ogawa H, Suefuji H, Takazoe K, Soejima H, Sakamoto T, Miyamoto $\mathrm{S}$, et al. Difference in fibrinolytic activity between multivessel coronary spasm and one-vessel coronary spasm. Am J Cardiol 2000; 85: $98-101$.

29. Sugiishi M, Takatsu F. Cigarette smoking is a major risk factor for coronary spasm. Circulation 1993; 87: 76-79.

30. Lichtlen PR, Hugenholtz PG, Rafflenbeul W, Hecker H, Jost S, Deckers JW. Retardation of angiographic progression of coronary artery disease by nifedipine: Results of the International Nifedipine Trial on Antiatherosclerotic Therapy (INTACT). Lancet 1990; 335: $1109-1113$.

31. Waters D, Lesperance J, Francetich M, Causey D, Theroux P, Chiang YK, et al. A controlled clinical trial to assess the effect of a calcium channel blocker on the progression of coronary atherosclerosis. Circulation 1990; 82: 1940-1953.

32. Sueda S, Fukuda H, Watanabe K, Suzuki J, Saeki H, Ohtani T, et al. Magnesium deficiency in patients with recent myocardial infarction and provoked coronary artery spasm. Jpn Circ J 2001; 65: 643-648.

33. Yamagishi M, Miyatake K, Tamai J, Nakatani S, Koyama J, Nissen SE. Intravascular ultrasound detection of atherosclerosis at the site of focal vasospasm in angiographically normal or minimally narrowed coronary segments. J Am Coll Cardiol 1994; 23: 352-357.

34. Saito S, Yamagishi M, Takayama T, Chiku M, Koyama J, Ito K, et al. Plaque morphology at coronary sites with focal spasm in variant angina: Study using intravascular ultrasound. Circ J 2003; 67: 1041 1045

35. Emond M, Mock MB, Davis KB, Fisher LD, Holmes DR Jr, Chaitman BR, et al. Long-term survival of medically treated patients in the Coronary Artery Surgery Study Registry. Circulation 1994; 90: $2645-2657$.

36. Beltrame JF, Sasayama S, Maseri A. Racial heterogeneity in coronary artery vasomotor reactivity: Differences between Japanese and Caucasian patients. J Am Coll Cardiol 1999; 33: 1442-1452. 